\title{
Supporting Reasoning and Problem-Solving in Mathematical Generalisation with Dependency Graphs ${ }^{\star}$
}

\author{
Sergio Gutiérrez ${ }^{1}$, Darren Pearce ${ }^{1}$, Eirini Geraniou ${ }^{2}$, and Manolis Mavrikis ${ }^{2}$ \\ London Knowledge Lab ${ }^{1,2}$ \\ 1 Birkbeck College \{darrenp, sergut\}@dcs.bbk.ac.uk \\ 2 Institute of Education \{m.mavrikis, e.geraniou\}@ioe.ac.uk
}

\begin{abstract}
We present a brief description of the design of a diagrambased system that supports the development of thinking about mathematical generalisation. Within the software, the user constructs a dependency graph that explicitly shows the relationships between components of a task. Using this dependency graph, the user manipulates graphical visualisations of component attributes which helps them move from the specific case to the general rule. These visualisations provide the user with an intermediate representation of generality and facilitate movement between the specific details of the task, the appropriate generalisations, verbal descriptions of their understanding and various algebraic representations of the solutions.
\end{abstract}

\section{Introduction}

The need to recognise, express and justify generality is at the core of mathematical thinking and scientific enquiry. However, a voluminous body of mathematics education research [1] suggests that expressing generality, recognising and analysing patterns and, in particular, articulating structure is complex and problematic for students [2].

We are addressing this problem by developing a mathematical microworld [3] that aims to promote the learning of mathematical generalisation by encouraging students to connect their actions during activities with the need to express generality. The microworld will be part of a learning environment which will also contain components that are (a) able to provide personalised support adapted to students' construction processes and (b) foster and sustain an effective online learning community by advising learners and teachers as to which constructions of other students to view, compare, critique and build upon.

A first version of this microworld has been presented in [4]. Several pilot studies using the software have shown that although learners are able to use the software effectively and that it facilitates their reasoning processes, there is a need to highlight the dependencies between shapes and the expressions

* Work supported by project MiGen (TLRP e-Learning Phase-II, RES-139-25-0381). 
manipulated. Since diagrammatic representations have been shown to support the reasoning process [5] and they hold potential for facilitating transfer of skills across subject matter [6], the remainder of this paper presents a brief description of proposed improvements to the user interface that use dependency diagrams to address the need described above.

\section{Dependency Graphs}

This section presents the new on-screen representation for the relationships between objects in the system. Building on lessons learnt from previous versions [4], the goals are to make the relationships between shapes explicit, direct and unambiguous. A graph explicitly relates expressions and shapes. In this dependency graph both expressions and shapes are represented as nodes, while the edges of the graph represent the relationships between them. Expressions use iconvariables [4] to represent concepts such as 'width of a shape' (see Figure 1). This is a compelling alternative to using letters which has been shown to be confusing when learning algebra [7].

There are three types of nodes: shapes, shape attributes (e.g. width), and expressions (that define shapes: attributes have to be described by means of an expression, e.g. ' 4 ' or 'width of that shape +2 '). Accordingly, there are three types of edges: shape-parameter (these edges link a shape with its parameters; therefore, they are declarative links, as they show a relationship that is defined by the system and not the learner), expression-parameter (that link a parameter with an expression; they are assignment links, they give a value - either concrete or variable - to a parameter), and parameter-expression (similar to the former ones, they are also assignment links; in this case the parameter gives the value to the expression, but the expression can only be an icon-variable). A simple example can be seen in Figure 1. The height and the width of the rectangle on the right are based on the dimensions of the rectangle on the left; they are both two units larger. It can be seen that the links that connect the parameters with icon-variables (e.g. dotted line connecting the left shape's parameters with the icon-variables used in the expressions that define the right shape) 'cross' the boundaries of the expression. This effect distinguishes these links from the other two types (apart from the different drawing style) and highlights their importance in the generalisation process.

A major concern in explicitly displaying the dependency graph is that of cognitive overload and generally making the system difficult to use. This is not only a performance issue, but it has been shown that learners are able to focus only on a small number of objects on the screen at the same time [8]. To address this, we propose that shapes are always shown but the graph is not shown in full on the screen; only those parts of the graph relevant to the student are shown. This could be inferred from the position of the cursor so that the user chooses which nodes are important by moving the mouse cursor over them, and the rest of the graph is hidden until requested. The user can additionally select which 


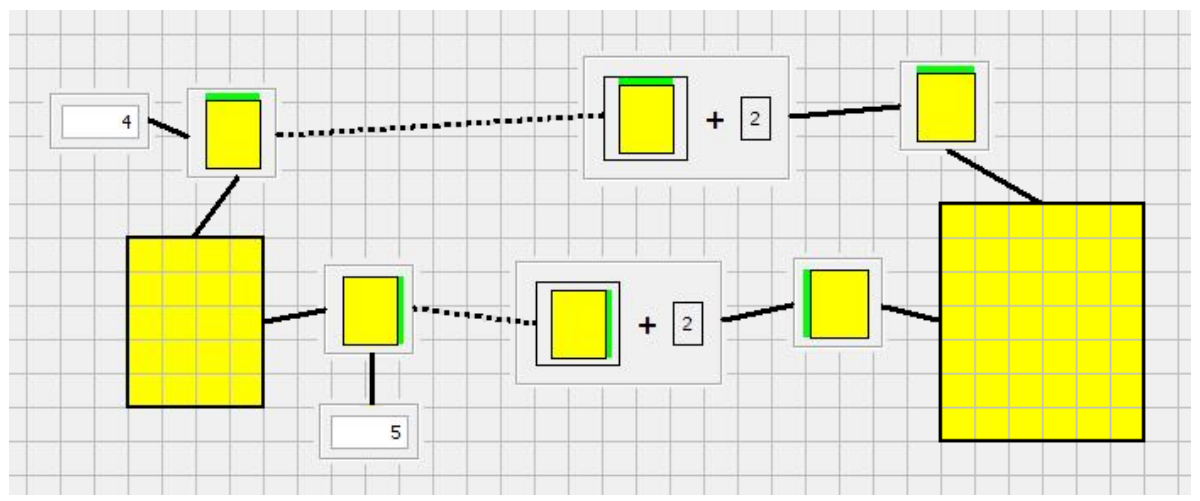

Fig. 1. A shape whose height and width depend on the dimensions of another shape.

parts of the graph they want to be able to see at all times. This affords the user full control over the degree to which the dependency graph is displayed.

An important feature of dependency graphs is not only the use of multiple representations for the students, but the visual links between them. When students construct new shapes based on their original shape, they can refer at any time to the connections between them. It must be noted that icon-variables are visually linked to the shapes from which they originate in a meaningful way (e.g. 'width' variables are connected to one of the horizontal lines of a rectangle).

From a pedagogical perspective, the importance of the use of a dependency graph is in giving students the opportunity to reason within their constructions. Students can see how their shapes are linked (or not) together and possibly be motivated to construct related shapes rather than looking for specific solutions. These graphs can be used as stepping stones in students' thinking before a general solution is reached, but also as justifications for their actions to themselves or their peers. By explaining their way of thinking, justifying how they reached their solutions and answering questions, they can demonstrate their understanding. Such actions could also be considered as a first approach to formal proof, since students try with such visual help to express generality and achieve reasoning 'in general' rather than 'in particular' cases.

The importance of this representation is that it helps students to make the shift from specific properties (i.e. numbers) to general relationships (by using icon-variables). Showing students creative representations (as well as symbolic) reinforces connections between them [9].

\section{Conclusions and Future Work}

We have presented a feature of a system that helps in the development of mathematical generalisation thinking. It clearly shows the relations that exist between several shapes. Studies using a former version of the software suggested that a 
more explicit relation between the attributes would help the learner to understand them and think in general terms (i.e. using variables instead of numbers). Dependency graphs are used to justify general rules and not just to describe them. Therefore, the system supports learners engaging in the process of mathematical generalisation by providing them with diagrams and representations that can be used as a resource for reasoning about generalisation. The system is diverted from the usual teaching method of algebra, in which students are introduced to letters that represent an unknown value and scaffolds the leap from the concrete to the general through icons that represent variables (e.g. 'width' of a shape).

Next step in the research involves performing studies with learners in order to assess the impact of the new approach as an assistance for the development of generalisational thinking. Different students learn in different ways and this multiplicity of representations can accommodate different kinds of learners. For example, visualisers might have an advantage when using this system. The impact of this representation on different types of learners demands further investigation.

\section{References}

1. Noss, R., Healy, L., Hoyles, C.: The construction of mathematical meanings: Connecting the visual with the symbolic. Educational Studies in Mathematics 33(2) (July 1997) 203-233

2. Mason, J., Graham, A., Johnston-Wilder, S.: Developing Thinking in Algebra. Paul Chapman Publishing (2005)

3. Balacheff, N., Kaput, J.: Computer-based learning environments in mathematics. In Bishop, A.J., Clements, K., Keitel, C., Kilpatrick, J., Laborde, C., eds.: International Handbook on Mathematics Education, Dordrecht: Kluwer (1996)

4. Pearce, D., Mavrikis, M., Geraniou, E., Gutiérrez, S.: Issues in the design of an environment to support the learning of mathematical generalisation (accepted). In: Proc. of the European Conference on Technology-Enhanced Learning. (2008)

5. Larkin, J., Simon, H.: Why a diagram is (sometimes) worth ten thousand words. Cognitive Science 11 (1987) 65-99

6. Novick, L., Hurley, S., Francis, F.: Evidence for abstract, schematic knowledge of three spatial diagram representations. Memory and Cognition 27 (1999) 288-308

7. Küchemann, D.: Algebra. In Hart, K.M., ed.: Children's Understanding of Mathematics, Antony Rowe Publishing Services (1981) 102-119

8. Moreno, R., Mayer, R.E.: Visual presentations in multimedia learning: Conditions that overload visual working memory. In Huijsmans, D.P., Smeulders, A.W.M., eds.: Visual Information and Information Systems, Springer-Verlag (1999) 793-800

9. Warren, E., Cooper, T.: The effect of different representations on year 3 to 5 students' ability to generalise. ZDM Mathematics Education 40 (2008) 23-37 